\title{
Priority Sequence of Generic Technologies Commercialization: Take Biotechnology for an Example
}

\author{
Shihui Wang, Rongfang Liu \\ School of Business Administration, South China University of Technology, Guangzhou, China \\ Email: shihui_wang92@163.com
}

How to cite this paper: Wang, S.H. and Liu, R.F. (2018) Priority Sequence of Generic Technologies Commercialization: Take Biotechnology for an Example. American Journal of Industrial and Business Management, 8, 735-746.

https://doi.org/10.4236/ajibm.2018.83050

Received: January 30, 2018

Accepted: March 25, 2018

Published: March 28, 2018

Copyright ( $\odot 2018$ by authors and Scientific Research Publishing Inc. This work is licensed under the Creative Commons Attribution International License (CC BY 4.0).

http://creativecommons.org/licenses/by/4.0/

\begin{abstract}
With the establishing and improving of generic technologies development system in various countries, the obstacles which block the development of generic technologies have been transferred from basic R \& D sessions to the downstream sessions, which refer to industrial applications. The research on integrating priority sequence of generic technologies commercialization in the stage of knowledge diffusion is desiderated. Given the limitation of generic technologies classification perspective based on bottom-up tree structure, using social network analysis method, this paper puts forward a method which identifying industrial generic technologies reversely by observing the sharing of proprietary technology to generic technologies from the top of technology tree, in order to pinpoint generic technologies with market conditions and those still need to be studied, moreover, to provide a scientific evaluation method for government intervention in the diffusion of generic technologies. In order to achieve this research, we take biotechnology as an example to conduct empirical test.
\end{abstract}

\section{Keywords}

Knowledge Diffusion, Generic Technology, Commercialize, Priority Sequence, Biotechnology

\section{Introduction}

Generic technologies promote China's strategic emerging industries to catch up with and surpass advanced world levels by providing technical platform support, which has great commercial value [1]. Due to the fundamentality, strategy, risk and externality of generic technologies and the spontaneous market forces, he- 
terogeneous market failures appear in various stages of generic technologies life cycle [2], which depends largely on the participation and supports of the government [3]. This paper puts forward a method which identifying industrial generic technologies reversely by observing the sharing of proprietary technology to generic technologies from the top of technology tree, in order to pinpoint generic technologies with market conditions and those still need to be studied, moreover, to provide a scientific evaluation method for government intervention in the diffusion of generic technologies.

Through the empirical study, the result shows that due to the market failure, on the one hand, although generic technologies with high centrality are located at core position of technical platform, its weak in technology diffusion. In addition, the diffusion channels of generic technologies are diversified and multi-layered, thus makes spread of generic technologies becoming more complicated. On the other hand, some of generic technologies have low generic-technology centralities but strong proprietary-technology support. Once the property rights and legal protection system is not perfect or not implemented, suppliers chose to block the technology in case technology innovations being spread excessively for free.

The main contribution of this paper is to propose a network method for selecting priority sequences of generic technologies, therefore provide a theoretical basis for the government to formulate science and technology policy. In addition, the findings from this paper could also be generated to technology spinout of companies. However, this article also has some limitations. The research studies generic technologies from the industry level, but the commercialization of generic technologies ultimately requires companies to practice. Consequently, how to integrate this generic technologies selection method into the enterprise technology spinout is one of the future research directions of generic technologies.

\section{Literature}

Scholars have done a detailed study on the government's role in the supply of generic technologies, mainly focused on generic technologies development [4], platform construction [5], innovation systems [6] etc. However, there is still a distance from the supply end to the diffusion end of generic technologies. As the core session for achieving its value, screening and identifying generic technologies, especially forecasting and rating precisely of its commercial potential, have recently become major issues in this field [7] [8] [9] [10].

With the establishment and improvement of generic technologies development system in China, the obstacles which block the development of generic technologies have been transferred from basic R \& D sessions to the downstream sessions, which refer to industrial applications. As a whole, it appears inefficient diffusion and inconspicuous spillover when generic technologies transferred to SMEs [11]. Due to the absence of the diffusion mechanism, especially for some leading enterprises participating in generic technologies $\mathrm{R} \& \mathrm{D}$ projects, it is 
difficult for other companies to share the results of generic technologies investment. In this circumstance, generic technologies thus turn out to be a substantive proprietary technology [12]. Owing to the tree structure of generic technologies [13], diffusion policy should be chosen based on commercial potential of generic technologies rather than be applied across the board.

Gambardella \& McGahan [8] suggest that the technology distance between generic technologies and derived proprietary technology is the key to achieve generic technologies commercialization. Technical boundaries are dynamic and ambiguous, while top of the tree structure is complex and networked [14], the quantitative researches which based on a bottom-up technology evolution perspective in the past had many limitations. For example, Delphi method is largely affected by subjective interference, therefore lack of certain objectivity and strong operability.

Given the limitation of generic technologies classification perspective based on bottom-up tree structure, using social network analysis method, this paper puts forward a method which identifying industrial generic technologies reversely by observing the sharing of proprietary technology to generic technologies from the top of technology tree, in order to pinpoint generic technologies with market conditions and those still need to be studied. Moreover, to provide a scientific evaluation method for government intervention in the diffusion of generic technologies.

\section{Theoretical Background}

As a platform for companies to research and develop proprietary technology, generic technologies broadly can be divided into basic generic technologies and applied generic technologies [2] [3] [12]. Basic generic technologies is the basic technology with domain characteristics and public properties, focusing on social supply. Applied generic technologies are the kind of system technology with clear industrial application goals. Because of its direct support for the development of enterprise proprietary technology, applied generic technologies have both public and private properties and is more embodied as a private property [15].

According to evolutionary theory of technology change, two generic technologies at the bottom of the technology tree structure have continued to extend upward, thus derived application-oriented and professionally oriented proprietary technology. Therefore, in general, technology shows a tendency to move forward [8] [13]. Many early studies have pointed out the role which government played at the supply end of generic technologies, especially in eliminating the market failure is positive. However, diffusion is the only way for generic technologies to produce economic benefits. It has been widely concerned by domestic and international academia and industry that the insufficient sharing of generic technologies during the derivation stage of proprietary technology was the result of delayed commercialization process [7] [16] [17]. Recent studies 
shows that economy, institution, legal, behavior and other non-technical elements have restricted the barriers to transform, which led to the diffusion of generic technologies obviously 1) much smaller than expected, such as biotechnology industry [8]; 2) much slower than expected, such as nanotechnology, solar thermal utilization technology industry [18] [19] [20].

It has been provided systematic policy implications for researches about technology diffusion related issues based on a bottom-up tree structure of technology evolution perspective. However, technical boundaries are dynamic and ambiguous, while top of the tree structure is complexity and networked [14], thus make the technical nature presents a mixed state during the process of government intervention, which is difficult to clarify [21]. For this reason, commercialization of generic technologies cannot be evaluated and fed back effectively. Consequently, this paper starts with the proprietary technology at the top of the technology tree structure, then deduces the commercial prioritization of underlying generic technologies based on reverse innovation logic and network algorithm.

\section{Methodology}

1) Industry

Emerging industries often come from emerging technologies. As an emerging industry, biotechnology industry network is much larger. While being rich in resources, it also means an increase in uncertainty. The development of such industries often depends on technology improvement. Raija et al. suggest that highly cited patents often become an important symbol of emerging industries development. Ahuja, Gregory and Wilfred propose that the difference between highly cited patents and general patents are mainly reflected in technical impact. The cited times of a patent is the most direct indicator of technical impact. As Trajtenberg once suggested in his paper, if a patent is heavily cited, it shows that this patent has been widely used in other technical fields, thus has great value and higher quality. Therefore, this article uses highly cited patents on behalf of major inventions. On the other hand, generic technologies are a kind of technology that has already been or may be universally applied in many fields. Its research results can be shared and have deep impact on the entire industry or across multiple industries and the companies belong to them.

2) Data

The data used for the empirical analysis is the Derwent Innovations Index (DII) database. According to the existing research, patents citation has a lag period of three or five years. In order to ensure that the patents are fully cited to reflect the sharing of proprietary technologies on generic technologies, this paper choose five-year lag period of patent citation. Therefore, the study period is set in 2008 to 2012 and the data was downloaded in October, 2017. The WOS (Web of Science) database provides CrossSearch. With unique citation retrieval mechanism and powerful cross-search function, crossed citation across Derwent 
(DII) patent database and three citation index databases (SCI, SSCI \& AHCI) is constructed. It is constantly updating at a rate of tens of thousands per week, including data from 1963 to present. Each of the patent document in the DII database cites three different classification codes, i.e. IPC codes, DC codes and MC codes. Besides the codes, DII database also covers patent information of multiple countries. The comprehensive data information has an advantage over observing the development process of emerging technology.

This paper has opted to ground our DII research strategy on the OECD definition of biotechnology, which covers the following IPC classes: A01H1/00, A01H4/00, A61K38/00, A61K39/00, A61K48/00, C02F3/34, C07G11/00, C07G 13/00, C07G15/00, C07K4/00, C07K14/00, C07K16/00, C07K17/00, C07K19/00, C12M, C12N, C12P, C12Q, C12S, G01N27/327, G01N33/53*, G01N33/54*, G01N33/55*, G01N33/57*, G01N33/68, G01N33/74, G01N33/76, G01N33/78, G01N33/88, G01N33/92. Those codes with * also include their sub-codes, e.g. in code G01N33/53*, the sub-codes, i.e. G01N33/532, etc. are also included.

Through the above method, this article has downloaded 47,207 patents in the biotechnology industry for the five years from 2008-2012. The top three cited frequency are $261,183,181$ times. We created the basic patents database by choosing highly cited patents (top 5\%) which were cited more than 38 times. The basic patents database comprises 212 patents, which includes 14,228 previously cited patents. Apparently biotechnology patents are generally with high technical content, thus those 212 highly cited patents are representative. This article tracks the 212 patents and their citations, which can observe the priority sequence of generic technologies commercialization as well as reflect the knowledge transfer of generic technologies in biotechnology industry.

\section{3) Method}

There is relatively few research on the quantitative analysis of generic technologies identification. The majority research mainly takes the patent data as an example. Based on the analysis of the references between technologies, they referred to set relevant identification index in order to select and identify generic technologies in related fields. P. Moser \& T. Nicholas defined the way technology general degree is calculated by referring to the calculation of the HHI index along with analyzing the citations of patents. Furthermore, distinguishing whether a technology is a generic industry technology by using the technology general degree index. Luan Chunjuan [22] introduced a pure quantitative analysis method to identify industrial generic technologies. She suggests that if there is a co-occurrence relationship between $\mathrm{A}$ and other technical fields, $\mathrm{A}$ is called a generic technology field. The more prominent and close this co-occurrence is, the larger and deeper its scope of application and impact is. Based on this hypothesis, combined with the classification features of DII patent data, Luan Chunjuan applies co-occurrence analysis methods to generic technologies measurement for the first time. In addition to that, she tries to identify indices like technology co-occurrence rate, betweenness centrality, multi-measurement centrality, 
technology-related degree etc. according to the definition of industrial generic technologies, then conducts the empirical study of the above indices taking solar energy technology as an example. Luan Chunjuan's methods mainly focus on the quantitative study of patent data. She believes that, in order to guarantee the accuracy and validity of generic technologies identification, the combination of quantitative and qualitative analysis is needed for getting a better result of generic technologies choosing.

Co-occurrence analysis is based on the co-word analysis and co-cited theory clustering, comparing and analyzing the literatures that have direct relevance in the content, thus identifying and extracting valuable information from it. Co-occurrence analysis was first proposed by linguists and applied in the analysis of semantic closeness. If two terms occur frequently together in a certain order under a single context, it means they have high semantic closeness. After that, co-occurrence analysis is widely used in the study of similarity, including co-authored analysis, co-cited analysis, co-word analysis and co-classified analysis etc. Co-cited analysis is mainly used in the field of research and exploration of emerging technology, while co-word analysis is applied to the forefront of research and the detection of leading technology analysis. Although structured methods of integrating patent analysis and text analysis help to reveal the extent and path of knowledge flows within the industry, the existing framework still has some defects, mainly reflected in the respect that the patent citation information system is not complete and low digging. Tapping patents information by measuring textual similarity between basic patents and the knowledge they used or spread can effectively capture the degree of knowledge transfer between basic and referenced patents.

Based on a bottom-up tree structure of generic technologies classification perspective, this paper proposed a set of structured methodology that bridges the use of knowledge and the process of knowledge dissemination, which consists of the following five steps:

The first step, creating the basic patents database by choosing highly cited patents which largely represent high quality of patents and intensive flow of knowledge. For the above-mentioned reasons, highly cited patents are more suitable to detect the knowledge flow of generic technologies. Both the basic patents and cited patents are from DII database. Basic patents are not only the key node that distinguish between cited patents and citing patents, but also the main carrier of knowledge diffusion. Therefore we regard basic patents as research objectives.

The second step, extracting the abstracts of the basic patents and cited patents to build the basic patents and the cited patents co-word network respectively, so that we can tap the inherent characteristics of knowledge flow. The abstract is the condensation of the main contents of the patent application. It provides more comprehensive and richer information compared to its title. Among them, the descriptor list is standardized to delete variants of the same word.

The third step, conducting technical discipline classification according to DC 
codes in order to build technology disciplines network. Calculating the centrality of DC codes on the basis of relevant theories and calculation methods of betweenness centrality. The technical field or technical theme represented by "bridge" often has functions of integration, driving and radiation, which is able to cultivate new growth points and be regarded as the key technical field or the key technical theme. The degree centrality can be used to measure a single core technological field or core technological theme. Measuring the core technological group of a complex technological network is more appropriate to choose the eigenvector centrality index.

The forth step, based on the key words extracted from the basic patent, the knowledge flow degree of different technical disciplines is measured by the textual similarity which is obtained through the cosine algorithm. DC codes are the technological classification codes quoted by Derwent technical experts as well as implementing the unified classification standard of one letter and two numbers. Whether implementing the unified classification standard or the overall quantity, DC codes are suitable for analysis of technical classification. Extracting keywords from basic patents and cited patents in order to calculate cosine similarity-a similarity measurement that can understand and calculate long vectors and sparse vectors easily. The cosine similarity is defined as follows:

$$
\operatorname{Sim}(P, C)=\frac{\sum_{k=1}^{n} P_{k} C_{k}}{\sqrt{\sum_{k=1}^{n} P_{k}^{2} \sqrt{\sum_{k=1}^{n} C_{k}^{2}}}}
$$

where $P_{k}$ refers to the frequency of keyword $k$ in basic patents; $C_{k}$ refers to the frequency of keyword $k$ in citation patents at patent-class level. If keywords do not exist in the corresponding patents, $P_{k}=0$, otherwise, $P_{k}=1$. Similarly, if keywords do not exist in the corresponding citied/citing patents, $C_{k}=0$, otherwise,, $C_{k}=1 . n$ refers to the quantity of patent-class for basic patents and cited/citing patents.

The fifth step, calculating the centrality and similarity of each DC code and then obtaining the priority sequence according to the ranking of arithmetic values.

\section{Results}

As for relationship network, centrality measurement is a way to describe the "right" of actors in a network. If an actor is directly connected to the others in its local environment, its eigenvec centrality is high. The more frequently a patent is cited, the more widely its technical field is applied to other technical fields. The higher its centrality is, the closer its technical field is connected to others as well as the more apparent features of generic technologies in this field. This paper constructed network for co-occurrence relationships of technical disciplines. The network comprises of technical disciplines (nodes) that are linked by their co-occurrence relationships with other technical disciplines (edges). In addition to that, we calculated the eigenvec centrality following the Network-Centrality- 
Eigenvec centrality steps of UCINET. Then selecting the top 13 technical fields with values greater than 3.0 and considering them as generic technological fields in the biotechnology industry (Figure 1).

D16 has the highest eigenvec centrality, which is 100.247. It refers to fermentation industry which is a kind of new technique that produces useful products for humans using modern techniques as well as applying microorganism to industry procedures. B04 represent natural products and polymers, testing, compounds of unknown structure. It has the second largest number of co-occurrence partners with the eigenvec centrality of 91.76 . S03 refers to scientific instrumentation, photometry, calorimetry. In a word, applications of biotechnology in engineering. Most of the other generic technologies fields also locates in the center of the technical network and have co-occurrence relationship with many technical fields. For example, C06-biotechnology, plant genetics, veterinary vaccines; A96-medical, dental, veterinary, cosmetic; J04-chemical/physical processes and apparatus including catalysis.

When conducting text analysis, this paper used co-words analysis program Citespace. By the means of extracting the keywords from abstracts, 37 keywords were extracted from the basic patent (Figure 2). Because the software unable to

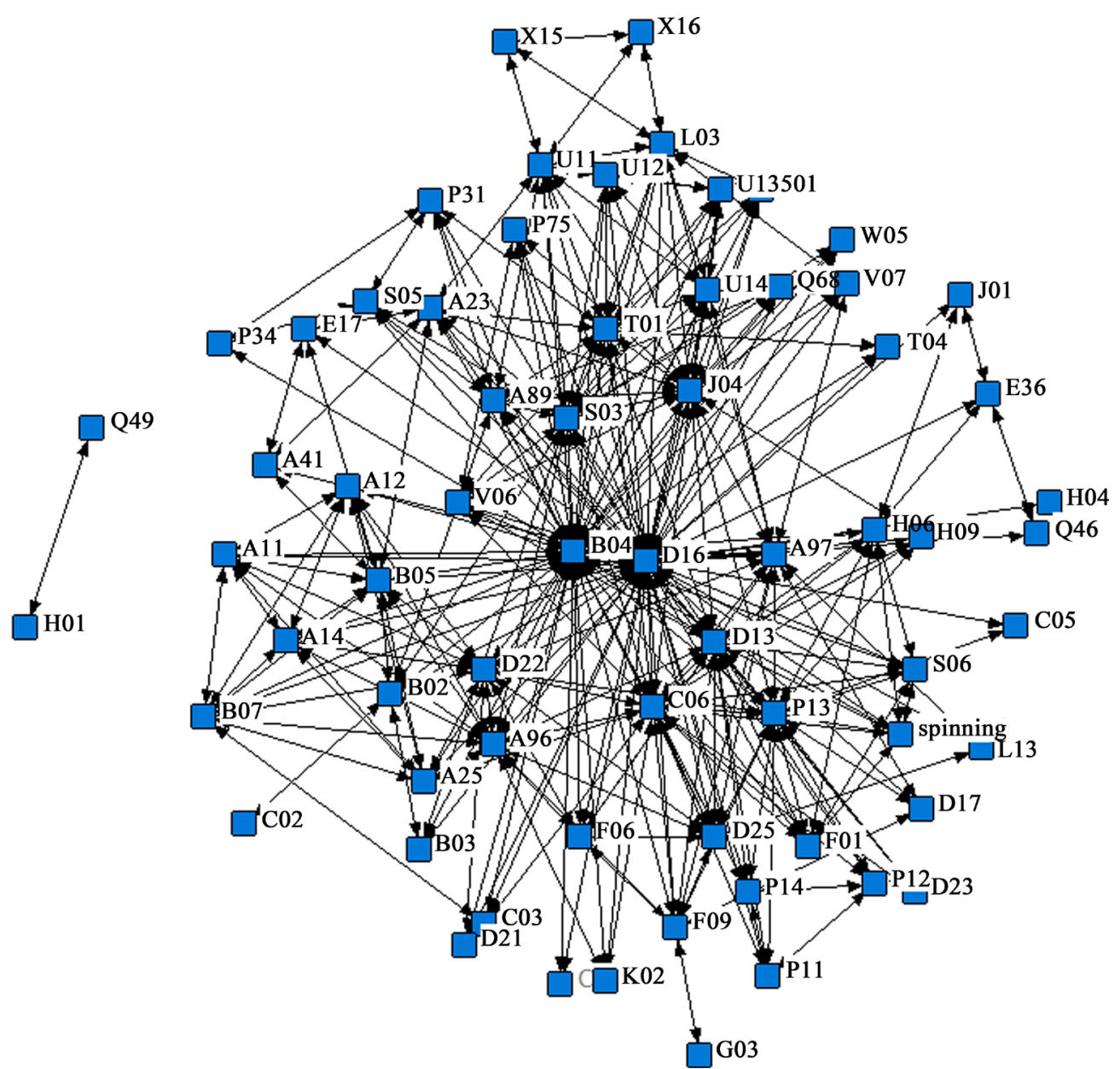

Figure 1. Betweenness network for DC codes of basic patents. 

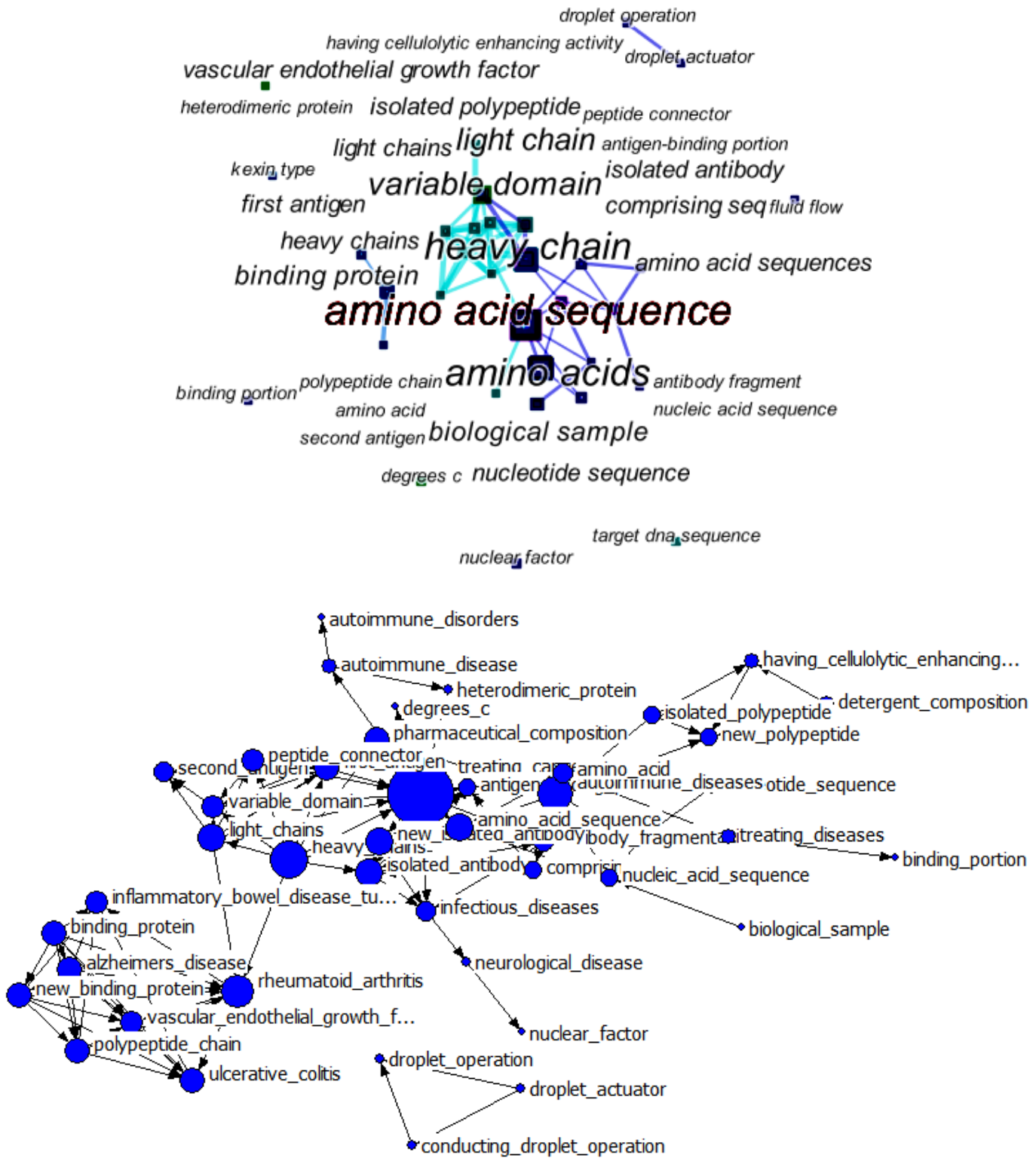

Figure 2. Co-occurrence network of keywords.

interpret the context or meaning, some irrelevant or unimportant words may be extracted. Further, combined with experts' advice on filtering keywords manually, 33 keywords were selected as keywords set of this article. Likewise, we extracted 1220 keywords from cited patents. It is easy to find that the keywords in B16 and B04 which are at the core position of technical disciplines are located in the center of the co-word network. For instance, amino acid sequences, detergent composition, etc. Besides, keywords in genetic engineering field, such as treating cancer, antibody fragment, coding sequence, DNA molecule etc. are also located at the core position of co-word network. In a word, there are different evolutionary directions for the research of technical disciplines and biotechnology. The top 10 DC codes of the priority sequence of generic technologies commercialization in biotechnology industry are C06, S03, P13, A96, F09, B05, D13, 
Table 1. Centralities, similarities and products of DC codes in biotechnology industry.

\begin{tabular}{ccccc}
\hline DC codes & Centrality & Similarity & Centrality * Similarity & Priority sequence \\
\hline C06 & 2.894 & 59.24239101 & 171.4475 & 1 \\
S03 & 2.042 & 40.50508166 & 82.71138 & 2 \\
P13 & 1.728 & 29.69798667 & 51.31812 & 3 \\
A96 & 0.761 & 56.23079034 & 42.79163 & 4 \\
F09 & 2.974 & 14.35685106 & 42.69728 & 5 \\
B05 & 0.779 & 45.67713355 & 35.58249 & 6 \\
D13 & 1.757 & 19.84577469 & 34.86903 & 7 \\
J04 & 1.719 & 15.89968462 & 27.33156 & 8 \\
A97 & 0.906 & 26.81110459 & 24.29086 & 9 \\
T01 & 0.77 & 28.32878161 & 21.81316 & 10 \\
\hline
\end{tabular}

J04, A97, T01 ${ }^{1}$. The centralities, similarities and products are shown in Table 1.

\section{Conclusions and Recommendations}

This paper introduces a network analysis that integrated citation analysis and text mining. The paper also puts forward a method which is able to identify industrial generic technologies reversely by observing the sharing of proprietary technology to generic technologies from the top of technology tree, in order to pinpoint generic technologies with market conditions and those still need to be studied, moreover, to provide a scientific evaluation method for government intervention in the diffusion of generic technologies.

The empirical results of biotechnology industry show that, although generic technologies with high centrality are located at core position of technical platform, it's weak in technology diffusion. The main reason leading to this phenomenon is due to the market failure in the process of fundamental generic technologies diffusion. Technical recipient still needs to do a lot of secondary development work, which lead to the diffusion of technology licensing, technology transfer and others are limited under market mechanisms. In addition, the diffusion channels of generic technologies are diversified and multi-layered. Different combinations at all levels will produce diverse effects of proliferation, thus makes spread of generic technologies becoming more complicated. On the other hand, according to the empirical results, some of generic technologies have low generic-technology centralities but strong proprietary-technology support. It is largely results from the market failure in applied generic technology. Once the property rights and legal protection system is not perfect or not implemented,

${ }^{1}$ C06: biotechnology, plant genetics, veterinary vaccines; S03: scientific instrumentation, photometry, calorimetry; P13: plant culture, dairy products; A96: medical, dental, veterinary, cosmetic; F09: paper-making production of cellulose, chemical treatment of wood; B05: other organics-aromatics, aliphatic, organo-metallics; D13: other foodstuffs and treatment; J04: chemical/physical processes and apparatus including catalysis; A97: miscellaneous goods not specified elsewhere; T01: digital Computers. 
suppliers chose to block the technology in case technology innovations being spread excessively for free.

On the basis of the problems we found, this paper provides following suggestions:

First of all, China should perfect the technology sharing mechanism. By completing intermediary services, strengthening infrastructure construction and overcoming the drawbacks of information asymmetry in the diffusion mechanism, thereby promoting the diffusion and sharing of generic technologies among industries and regions. In addition, $\mathrm{R} \& \mathrm{D}$ supply of generic technologies requires input from other resources. Therefore, in accordance with the requirements of the level of investment in technology in innovative countries, the government should increase its financial support and establish a special fund to support China's plans of action on basic generic technologies.

Secondly, generic-technology platform is a breakthrough for its development. The government should strengthen its leading force in the $\mathrm{R} \& \mathrm{D}$ process of generic technologies in order to build a platform system for innovation as well as to intensify the relationship among institutions, universities and enterprises. Furthermore, the government should actively guide the platform to devote itself to innovation on industrial generic technologies along with providing generic-technology support for the development of proprietary technology, so that to ensure the effective operation of industrial generic-technology innovation system.

\section{References}

[1] Slayton, R. and Spinardi, G. (2016) Radical Innovation in Scaling up: Boeing's Dreamliner and the Challenge of Socio-Technical Transitions. Technovation, 47, 47-58. https://doi.org/10.1016/j.technovation.2015.08.004

[2] Tassey, G. (2004) Underinvestment in Public Good Technologies. Journal of Technology Transfer, 30, 89-113. https://doi.org/10.1007/s10961-004-4360-0

[3] Li, J.Z. (2006) Industrial Generic Technology: Concept, Category and System Supply. Forum on Science and Technology in China, 3, 45-47.

[4] Xu, D. and Xu, F. (2010) Research on the Method of Defining and Selecting Industrial Generic Technology: From the View of Management of S \& T Programs. China Soft Science, 4, 73-79.

[5] Zhao, H., Li, J. and Wei, H.Z. (2015) The Innovation Mode of Generic Technology in the Industry Cluster: From the Perspective of Enterprise Contribution. Science Research Management, 36, 53-59.

[6] Cao, L.C. and Yang, S.L. (2005) Research on the Construction of Industrial Generic Technology Innovation System. China Soft Science, 11, 77-82.

[7] Ardito, L., Petruzzelli, A.M. and Albino, V. (2015) From Technological Inventions to New Products: A Systematic Review and Research Agenda of the Main Enabling Factors. European Management Review, 12, 113-147. https://doi.org/10.1111/emre.12047

[8] Gambardella, A. and Mcgahan, A.M. (2010) Business-Model Innovation: General Purpose Technologies and Their Implications for Industry Structure. Long Range Planning, 43, 262-271. https://doi.org/10.1016/j.lrp.2009.07.009 
[9] Gambardella, A. and Giarratana, M.S. (2013) General Technological Capabilities, Product Market Fragmentation, and Markets for Technology. Research Policy, 42, 315-325. https://doi.org/10.1016/j.respol.2012.08.002

[10] Maine, E. and Garnsey, E. (2006) Commercializing Generic Technology: The Case of Advanced Materials Ventures. Research Policy, 35, 375-393. https://doi.org/10.1016/j.respol.2005.12.006

[11] Cogez, P., Kokshagina, O., Masson, P.L., et al. (2015) Industry-Wide Technology Road Mapping in Double Unknown-The Case of the Semiconductor Industry. IEEE International Conference on Engineering, Technology and Innovation, Jun 2013, The Hague, 1-13.

[12] Li, J.Z. (2011) Supply and Diffusion Model Selection of Generic Technology. Science of Science and Management of S. \& T., 32, 5-12.

[13] Bresnahan, T.F. and Trajtenberg, M. (1995) General Purpose Technologies "Engines of Growth"? Journal of Econometrics, 65, 83-108. https://doi.org/10.1016/0304-4076(94)01598-T

[14] Baykara, T., Özbek, S. and Ceranoğlu, A.N. (2015) A Generic Transformation of Advanced Materials Technologies: Towards More Integrated Multi-Materials Systems via Customized R \& D and Innovation. Journal of High Technology Management Research, 26, 77-87. https://doi.org/10.1016/j.hitech.2015.04.008

[15] Tassey, G. (2008) Modeling and Measuring the Economic Roles of Technology Infrastructure. Economics of Innovation \& New Technology, 17, 615-629. https://doi.org/10.1080/10438590701785439

[16] Datta, A., Mukherjee, D. and Jessup, L. (2015) Understanding Commercialization of Technological Innovation: Taking Stock and Moving Forward. $R \& D$ Management, 45, 215-249. https://doi.org/10.1111/radm.12068

[17] Li, Q., Maggitti, P.G., Smith, K.G., et al. (2013) Top Management Attention to Innovation: The Role of Search Selection and Intensity in New Product Introductions. Academy of Management Journal, 56, 893-916. https://doi.org/10.5465/amj.2010.0844

[18] Maine, E. (2015) Scientist-Entrepreneurs as the Catalysts of Nanotechnology Commercialization. Social Science Electronic Publishing, 2, 301-308.

[19] Capozzi, M.M., Gregg, B. and Howe, A. (2010) Innovation and Commercialization, 2010: McKinsey Global Survey Results. McKinsey Co., New York.

[20] Philibert, C. (2006) Barriers to Technology Diffusion: The Case of Solar Thermal Technologies. IEA, Paris.

[21] Taebi, B., Correljé, A., Cuppen, E., et al. (2014) Responsible Innovation as an Endorsement of Public Values: The Need for Interdisciplinary Research. Journal of Responsible Innovation, 1, 118-124. https://doi.org/10.1080/23299460.2014.882072

[22] Luan, C.J. (2015) Generic Technology Measurement System and Application. Science Press, Beijing. 